Part IV. Gravitation Theory and Fundamental Interactions

\title{
ISOLATED SYSTEMS IN GENERAL RELATIVITY
}

\author{
Jürgen Ehlers* \\ Max-Planck-Institut für Physik und Astrophysik \\ 8000 Munich 40 \\ Federal Republic of Germany
}

\section{INTRODUCTION}

Almost all quantitative information about the gravitational interaction has been obtained from studies of nearly isolated systems composed of a few macroscopic bodies such as the solar system or double stars, and in the foreseeable future clean, precise tests of gravity-theories are expected to be based exclusively on observations of such systems, too, as can be seen, e.g., from the lectures of J.H. Taylor and C.M. Will at this conference.

At present Einstein's general theory of relativity (GR) still appears to be the most successful theory of gravity. Therefore it is important to have a reliable mathematical description of isolated systems based on that theory.

It appears to be widely believed that the implications of GR for systems of slowly moving, well-separated and therefore weakly-coupled bodies-even bodies with strong self-fields such as neutron stars-have been deduced satisfactorily with an accuracy sufficient to calculate not only quasistationary, first post-Newtonian effects of order $(\mathrm{v} / \mathrm{c})^{2} \sim \mathrm{Gm} / \mathrm{rc}^{2}$ like "anomalous" perihelion advances, but even the dominant, secular gravitational radiation reaction effects on the orbits, of order $(v / c)^{5}$. Such calculations have been applied, e.g., to the binary system containing the pulsar PSR $1913+16$. A minority of relativity-theorists including the present author does not share this opinion, for reasons given below, and in references 1-4 and the papers and books quoted therein.

If one argues by analogy with Newtonian mechanics and Maxwellian electrodynamics one obtains "plausible" results concerning, e.g., gravitational radiation damping. One can derive from a linearized version of the GR-field equation Einstein's quadrupole formula ${ }^{5}$ for the gravitational-radiation power loss, and equate that power loss to the rate of decrease of the Newtonian total energy expressed in terms of Keplerian elements, considered as secularly changing due to perturbing, gravitationalradiation reaction forces. ${ }^{6.7}$ The main shortcoming of this and similar arguments and calculations is, in my opinion, not that they employ approximations which have not been rigorously mathematically justified - that they share with many approximations used in physics - but rather that they:

1) employ notions which are not well defined in terms of basic concepts of GR, such as "gravitational field energy," "total mass and linear momentum" of a gravitationally bound body interacting with other such bodies, "point particle," "gravitational radiation reaction force," "near zone," "radiation zone";

2) use laws which have not been established within GR, such as an "energy balance between radiation and material sources";

\footnotetext{
*I dedicate this paper to my son Martin Ehlers.
} 
3) depend essentially on ad hoc assumptions which not only are without foundation within GR itself, but for which there are indications that they may be incompatible with the fundamental assumptions of GR or with each other, such as global coordinate conditions, particular global splittings of the metric into a flat background and a "small" perturbation, noncovariant "outgoing radiation conditions," negligibility of various kinds of "small" terms, etc. (For discussions of some of these difficulties and proposals to overcome them see references 3,4 , and 8-12).

It seems to me to be an important challenge to find derivations of observable relativistic effects, particularly structure and radiation effects, of isolated systems which are free of such shortcomings, and which are not based on mere analogies, however plausible, with Newton's or Maxwell's theory. Needed are, in particular, approximation methods which have been rigorously justified at least in theories simpler than Einstein's, and which permit if not an error estimate, at least a reasonable guess about error bounds.

In the following pages I shall describe some problems of the relativistic theory of isolated systems, hoping to stimulate more research in this area. As will be seen, some progress has been made in this field since the last Texas symposium, but the main questions remain unanswered. Classical GR is not a closed subject; it poses difficult, accessible problems related to real observations.

\section{ISOLATED SYSTEMS}

A general-relativistic model of an isolated system is an asymptotically flat $\dagger$ spacetime $\left(M, g_{\mathrm{ab}}\right)$ with a physically reasonable stress-energy-momentum tensor $T^{\mathrm{ab}}$ satisfying the Einstein-Hilbert equation

$$
G^{\mathrm{ab}}=T^{\mathrm{ab}},
$$

and hence also the local law of motion

$$
T_{: b}^{\mathrm{ab}}=0 .
$$

(Here I take $c=1,8 \pi G=1$, and choose the signature (+--) for the metric.) If the system consists of $N$ bodies separated by empty space, $T^{\mathrm{ab}}$ has to vanish outside of $N$ timelike, connected, spatially compact world tubes.

Such an isolated system may contain incident as well as outgoing gravitational radiation. If, in some interval of advanced time, the system is not subject to incident radiation or, more generally, if such radiation is not in resonance with a mode of the system, then presumably the emission of radiation will be accompanied by a damping of the motion of its material sources. (Asymptotically for " $t \rightarrow+\infty$ ", damping may already be implied by spatial and null asymptotic flatness since the system then has a finite total ADM-energy momentum, part of which is radiated to future null infinity. For simple model theories such a behaviour has been deduced. ${ }^{14}$ In GR a correspond-

†Instead of asymptotic flatness one can require $\left(M, g_{\mathrm{sb}}\right)$ to fit asymptotically into a cosmological model, ${ }^{13}$ a possibility which appears not to have been investigated extensively.

\$In this mechanical model the bodies are idealized as having boundaries, and contributions of nongravitational radiation to $T^{\mathrm{ab}}$ are not taken into account. Such contributions could be added, but are of secondary importance to celestial mechanics. 
ing theorem has not been rigorously formulated, let alone been proved.) The presence or absence of incident and emitted radiation can be expressed in terms of the asymptotic behavior of the gravitational field near past and future null infinity, respectively. .1,12,15 $^{2}$

Proposals concerning the precise meanings of the terms "asymptotically flat," "physically reasonable $T^{\mathrm{ab}}$," "condition for absence of incident radiation" will be mentioned below. We cannot, however, be sure that any of such proposed definitions are adequate until we know that models of isolated systems with the stipulated properties do, in fact (in a mathematical sense) exist and are sufficiently general to describe those physical situations for which the concepts have been invented. It appears that we are still far from that kind of knowledge. (The degree of understanding in this field bears some similarity to that in quantum field theory: There are reasonable proposals for the ground rules ("axioms"), a few exact results, no existence theorems of sufficient generality; but in spite of this various ill-founded approximation methods, plagued by infinities and other difficulties and not related to the exact parts of the theory, give remarkably good agreement with observations.)

Before discussing later in this paper the problem of constructing models of isolated systems-a task which up to now can be tackled by formal approximation methods only, except for one-body problems-I shall consider two topics which have been treated within the exact theory: the description of bodies as a whole and the derivation of their overall laws of motion, and the asymptotics of gravitational fields near various parts of infinity. The concepts, results and methods of these investigations would seem to be needed, at least in principle, before the main, global problem of constructing isolated systems can be treated rigorously, and they may also help in improving approximation methods.

\section{BODIES AND LAWS OF MOTION}

In order to represent mathematically the bodies (planets, stars, black holes) of an isolated system in GR one has to specify either the stress-energy-momentum tensor $T^{\mathrm{ab}}$ in equations 1 and 2 or the parts of the event horizon which bound the black holes.

In the following it will be assumed, as part of the "physical reasonableness" of $T^{\mathrm{ab}}$, that $T_{\mathrm{b}}{ }^{\mathrm{a}}$ maps the interior of each half of the null cone into itself, a condition which implies that for all local observers the energy density $T^{00}$ is positive, the magnitude of the density of linear momentum is less than $T^{00}$, and the stresses are also dominated by $T^{\infty}$.

The model of a point particle supported by a timelike world line $z(t)$, given formally by

$$
T^{\mathrm{ab}}(x)=m \int \dot{z}^{\mathrm{a}} \dot{z}^{\mathrm{b}}\left(-g g_{\mathrm{cd}} \dot{z}^{\mathrm{c}} \dot{z}^{\mathrm{d}}\right)^{-1 / 2} \delta(x, z) \mathrm{d} t,
$$

is incompatible with the field equation 1. (There does not exist a solution of (1)

$\S$ By "formal approximation methods" I mean methods for which it is not known whether they do, in fact, provide approximate solutions to any well-defined problem of the full theory, due to lack of convergence proofs, error estimates, etc. 
representing a spherically symmetric gravitational field generated by a distributional source (3). If (3) is used formally in approximation methods to solve (1), the metric not only diverges at the particle and thus makes equation 3 mathematically meaningless, but in addition has the wrong signature near the particle, even in linear approximation. Disregarding these trivial facts leads to spurious difficulties which may obscure the real problems. Using the word "renormalization" is no cure if such a procedure is not carried out beyond the linear approximation. IT The theorem that equations 2 and 3 , together with the assumption that $g_{\mathrm{ab}}$ is a $C^{\prime}$ Lorentz metric in a neighborhood of the particle's world line, imply the geodesic law, is by itself not relevant for GR since its assumptions contradict equation 1. A derivation of the geodesic law has to be based on assumptions which are compatible with (1), not only with (2). Essential steps towards such a derivation are contained in references $16-19,32$ ). Therefore ordinary bodies (as opposed to black holes) have to be represented in classical GR primarily as extended, deformable structures, that is, connected, spatially compact submanifolds with timelike boundary hypersurfaces, as has been emphasized by Fock, ${ }^{24}$ Dirac, ${ }^{20}$ Bondi, ${ }^{21}$ Chandrasekhar ${ }^{22}$ and others. Of course, one would like to describe bodies for most purposes of celestial mechanics by a world line and a few parameters, but the possibility and nature of such a simplified, approximate model has to be deduced from the primary model since ad hoc assumptions like (3) do not work.

I remark in passing that a Newtonian mass point with its gravitational field, represented in the spacetime formulation of Newtonian theory, ${ }^{23}$ can be obtained as an (exact) Newtonian limit ${ }^{25}$ of the Kerr or Schwarzschild spacetime for fixed mass and angular momentum parameter ${ }^{26}$ Combined with well-known black-hole theorems this indicates that the simplest "objects" of relativistic mechanics, corresponding to Newtonian mass points, are black holes. Some groundwork towards a theory of isolated systems of black holes has been done by Gibbons ${ }^{27}$ and D'Eath. ${ }^{19,28}$

Due mainly to the work of Dixon ${ }^{17}$ and some recent extensions of it by Ehlers and Rudolph $^{18}$ and by Schattner, ${ }^{29}$ a framework for the description of (ordinary) extended bodies in GR is available. For any such body, existence and uniqueness of a center-of-mass world line $l: z^{\mathrm{a}}(s)$ (contained in a convex hull of supp $T^{\mathrm{ab}}$ ) have been established $;^{29}$ a timelike linear momentum 4-vector $p^{\mathrm{a}}(s)$, an angular momentum bivector $S^{\text {ab }}(s)$ and a sequence of multipole moments $I_{\mathrm{n}}(s)$ have been defined covariantly ${ }^{17}$ on $l$ in terms of $g_{\mathrm{ab}}$ and $T^{\mathrm{ab}}$; and it has been shown ${ }^{17,18}$ that these dynamic body variables satisfy a finite system

$$
\begin{gathered}
\dot{z}^{\mathrm{a}}=\left(p_{\mathrm{b}} p^{\mathrm{b}}\right)^{-1 / 2} \cdot p^{\mathrm{a}}+V^{\mathrm{a}}\left(g_{\mathrm{bc}}, R^{\mathrm{b}}{ }_{\mathrm{cdc}} ; p^{\mathrm{b}}, S^{\mathrm{bc}}, F^{\mathrm{b}}, L^{\mathrm{bc}}\right) \\
\dot{p}^{\mathrm{a}}+\frac{1}{2} R_{\mathrm{bcc}}^{\mathrm{a}} \dot{z}^{\mathrm{b}} S^{\mathrm{cd}}=F^{\mathrm{a}} \\
\dot{S}^{\mathrm{ab}}+2 \dot{z}^{[\mathrm{a}} p^{\mathrm{b}]}=2 L^{\mathrm{ab}}
\end{gathered}
$$

of ordinary tensor differential equations. (The "force" $F^{a}$ and "torque" $L^{\text {ab }}$ depend on the multipole moments and the field $g_{\mathrm{ab}} \cdot V^{\mathrm{a}}$ measures the deviation of the CM-tangent vector $\dot{z}^{a}$ from the unit vector collinear with $p^{a}$ and is small for nearly spherical bodies. ${ }^{18}$ The explicit forms of $F^{\mathrm{a}}, L^{\mathrm{ab}}$ and $V^{\mathrm{a}}$ are given in references 17,18 . One has $p_{\mathrm{a}} V^{\mathrm{a}}=0, p_{\mathrm{a}} S^{\mathrm{ab}}=0$.) Conversely, the field $T^{\mathrm{ab}}$ can be reconstructed from $g_{\mathrm{ab}}$

IIn this connection, see also reference 68 . 
and the body variables $\left(z^{\mathrm{a}}, p^{\mathrm{a}}, S^{\mathrm{ab}}, I_{\mathrm{n}}\right)$, provided (4) holds; the reconstructed $T^{\mathrm{ab}}$ obeys (2). (See reference 17 and, for some mathematical extensions, see reference 30 .) The last two statements are particularly important since they show that the body variables describe the body completely, and that the equations 4 contain the same information as the local equation 2. Even the existence of such "global" body variables is remarkable. In the terminology proposed by Havas and Goldberg ${ }^{31}$ equations 4 are the "laws of motion" for extended bodies. They relate the evolution of the body variables to the gravitational potential $g_{a b}$ due (at least partly) to these bodies themselves. These laws are to be regarded as intermediate results on the way towards "equations of motion," viz. evolution equations containing body variables and, perhaps, nondynamic fields like a background metric only. (It is very questionable whether exact "equations of motion" exist in GR, in contrast to "laws of motion." I suspect that equations of motion exist as approximations only.)

The expressions $F^{\mathrm{a}}$ and $L^{\mathrm{ab}}$ in equations 4 are bilinear functionals of the multipole moments and certain "gravitational field strengths" derived from the metric $g_{\mathrm{ab}}$ and its connection. It is expected that these field strengths depend, within a particular body, mainly on that body itself and vary rapidly within the body, and that they depend only weakly on the other bodies of the system if the distances between the bodies are much larger than their sizes.

As in Newtonian mechanics and Maxwell-Lorentzian electrodynamics one would like to subtract self fields from the laws of motion (4) in such a way that the remaining external fields (for each body) vary only slowly within the body so as to permit a multipole approximation of the external forces and torques. Some self-field terms would be expected to represent radiation reaction forces, others might provide (finitely) "renormalized" variables $\hat{p}^{\mathrm{a}}, \hat{S}^{\mathrm{ab}}$. Such a program has not been carried out, but in view of the mentioned linearity it seems accessible, at least in conjunction with approximation methods to solve (1).

The Dixon-framework for the description of bodies in GR also offers a way to introduce simple body models such as "dynamically rigid"17,18 or "dynamically spherical" bodies, defined by suitable invariance properties of the system of multipole moments along the CM-world line $l$. Such body models might be useful relativistic analogs of Newtonian mass points or rigid bodies which avoid the inconsistencies inherent in combining (1) with (2).

Unfortunately, no possibility to combine the Dixon framework, which is based on (2) alone, with the field equation 1 has yet been suggested. Nevertheless, hitherto this approach is the only one in which dynamic body variables such as 4-momentum have been defined covariantly which obey various nontrivial, physically suggestive laws. (For further results see references 17, 18, 29, 30).

\section{ASYMPTOTICS}

Suppose a spacetime $\left(M, g_{\mathrm{ab}}, T^{\mathrm{ab}}\right)$ obeys the field equation (1) and contains a finite number of bodies as considered in the previous section. One would interpret it as a model of an isolated system only if its gravitational potential $g_{\mathrm{ab}}$ could be viewed as due to the bodies of the system (and, perhaps, some incoming radiation), and not to any outside matter. One cannot express this isolatedness by requiring, e.g. that $g_{a b}$ be 
the "retarded field of $T^{\mathrm{ab}}$ " since, 1) there are no Green functions associated with the nonlinear field equation 1, and 2) a "flat, nonMachian part" of the metric representing "pure inertia" would at any rate not be determined by $T^{\mathrm{ab}}$. It is, however, possible to formulate conditions on $\left(M, g_{\mathrm{ab}}\right)$ which express that the metric approaches that of flat spacetime "at infinity." Due to the basic work of Bondi, Sachs et al.," the geometrization and extension of their asymptotic description along future null cones to spacelike and past null infinity by Penrose, ${ }^{15}$ and extensive subsequent work by Geroch, Schmidt, Sommers, Ashtekar and others, there exists now an eleborate, flexible framework for describing in detail various kinds of asymptotic flatness. For systematic accounts and references see Geroch, ${ }^{11}$ Schmidt ${ }^{34.35}$ and Ashtekar and Hansen. $^{36}$ The last paper contains what is perhaps the most comprehensive notion of asymptotic flatness which has been proposed so far.

The key idea, due to Penrose, is to compress all distances "near infinity" by a conformal rescaling $g_{a b} \rightarrow \hat{g}_{a b}=\Omega^{2} g_{a b}$ of the physical metric $g_{a b}$, and to attach to the physical spacetime a boundary of "ideal events" at which both the conformal factor $\Omega$ and the unphysical metric $\hat{g}_{a b}$ remain well behaved, with $\Omega=0$, although $g_{a b}$ "becomes infinite" there. If this is possible (such that several technical conditions are satisfied ${ }^{11,35,36}$ ), the spacetime is said to be asymptotically flat.

The boundary of an asymptotically flat spacetime consists of future and past null infinity, called $\mathfrak{J}^{+}$and $\mathfrak{J}^{-}$, respectively, and spacelike infinity, $\mathfrak{I}^{0} . \mathfrak{J}^{+}$is the set of ideal end points of null geodesics (test signals) leaving the system; $\mathfrak{I}^{-}$, the set of ideal initial points of null geodesics incident upon the system; and, at least in the Kerr spacetime and its specializations, $g^{0}$ consists of a single point $i^{0}$, the common ideal end point of all spacelike geodesics, which also compactifies all spacelike, global Cauchy hypersurfaces of the physical spacetime. The union $\mathcal{J}^{+} \cup\left\{i^{\circ}\right\} \cup \mathcal{J}^{-}$forms the "null cone at infinity" with vertex $i^{0}$.

One merit of this extension of the physical spacetime by an ideal boundary is that the asymptotic behavior of fields at large distances from the material sources need not be described in terms of ill-definable limits, but can be described in terms of local properties at the boundary, like differentiability. A price one has to pay for this "finitization" is that one has to be very careful in the specification of the degree of differentiability of the conformal factor $\Omega$ and the unphysical metric $\hat{g}_{a b}$ at infinity, particularly at $i^{0}$, in order not to lose physically needed generality. (Differentiability at infinity reflects the rate at which fields in physical spacetime decrease. Simple analogy: A three times continuously differentiable function $f: \mathcal{R} \rightarrow \mathcal{R}$ has the property $f(x)=a+b x^{-1}+c x^{-2}+\mathcal{O}\left(x^{-3}\right)$ if the function $g: \mathcal{R}-\{0\} \rightarrow \mathcal{R}$ defined by $g(x)=f\left(x^{-1}\right)$ can be extended to $R$ such that the extended function has continuous derivatives up to the third order at 0 .)

An additional merit of the latest approach ${ }^{36}$ is that within it the point $i^{0}$ representing spatial infinity has a tangent space $T_{i}$ with a unique Lorentz metric $g_{a b}$. This tangent space provides a home for the Arnowitt-Deser-Misner 4-momentum $P_{0}{ }^{a}$, the coordinate-independent, intrinsic nature of which had remained somewhat obscure so far. Now $P_{0}{ }^{a}$ has gained the respectable status of a vector at $i^{0}$. What is more, $\|$ also the Bondi-Sachs-Penrose 4-momentum $P_{+}{ }^{a}(\Sigma)$, defined on cross-sections $\Sigma$ of future

|The following remarks are based on a preprint ${ }^{37}$ which became available only after the Texas symposium. They are included here since they partly answer an old question discussed in my talk, and permit me to simplify the subsequent remarks about total energy-momentum. 
null infinity (or $p_{-}{ }^{a}(\Sigma)$, at past null infinity), can be considered as a vector at $i^{0}$, so that $P_{0}{ }^{\mathrm{a}}$ and $P_{+}{ }^{\mathrm{a}}(\Sigma)$ can be meaningfully related to each other. ${ }^{37}$

$P_{0}{ }^{a}$ is interpreted as the total energy-momentum of the system, including all contributions from the gravitational field and the material sources. It is a timeindependent property of the state (= Cauchy data) of the system. On the other hand, the difference $P_{+}{ }^{a}\left(\Sigma_{1}\right)-P_{+}{ }^{a}\left(\Sigma_{2}\right)=: P_{R}{ }^{a}\left(\Sigma_{1}, \Sigma_{2}\right)$ represents the energy-momentum carried out of the system by gravitational radiation between outgoing null hypersurfaces ending at $\Sigma_{1}$ and $\Sigma_{2}$, respectively, with $\Sigma_{2}$ lying in the future of $\Sigma_{1}$. Thus, the function $\Sigma \rightarrow P_{+}{ }^{a}(\Sigma)$ informs about the evolution of the system.

Ashtekar and Magnon-Ashtekar have shown ${ }^{37}$ that if the 4-momentum carried by the gravitational waves emitted between the infinite past and some retarded instant $\Sigma$ is finite, then-as had been conjectured for some time- $\lim _{\Sigma \rightarrow i^{0}} P_{+}{ }^{a}(\Sigma)=P_{0}{ }^{a}$ holds, whence one can state the asymptotic energy-momentum balance

$$
P_{0}{ }^{\mathrm{a}}=P_{+}{ }^{\mathrm{a}}(\Sigma)+P_{\mathrm{R}}{ }^{\mathrm{a}}(\Sigma)
$$

valid for any cross section $\Sigma$ of $\mathfrak{J}^{+}$, i.e. for any retarded time. The 4-momentum radiated out before $\Sigma, P_{\mathrm{R}}{ }^{\mathrm{a}}(\Sigma)$, is known ${ }^{38,11}$ to be positive** or zero-gravitational waves carry positive energy. If $P_{+}{ }^{\mathrm{a}}(\Sigma)$, the 4-momentum "left over" at the instant $\Sigma$, were also positive, then, from (5), $P_{0}{ }^{a}$ would be positive, too, and (5) would imply that the total amount of energy that a system could possibly emit in gravitational waves could never exceed the total energy, $P_{0}{ }^{0}$.

Unfortunately, general conditions for $P_{+}{ }^{a}(\Sigma)$ to be positive have not been found, although the representation ${ }^{39}$

$$
P_{+}^{\mathrm{a}}(\Sigma)=\int_{\mathcal{H}} \tau^{\mathrm{ab}} \mathrm{d} V_{\mathrm{b}}
$$

of $\boldsymbol{P}_{+}{ }^{a}$ as a flux of the Landau-Lifshitz energy momentum complex $\boldsymbol{\tau}^{\mathrm{ab}}$ through a spacelike, asymptotically null hypersurface $H$ terminating at $\Sigma$, evaluated in special asymptotically orthonormal coordinates, as well as an inequality derived by Streubel ${ }^{39}$ suggest at least physically reasonable sufficient conditions for this to be the case.

On the other hand, general conditions have been found under which the ADMenergy, $P_{0}{ }^{0}$, is a strictly positive functional of asymptotically flat initial data supported by spacelike Cauchy hypersurfaces, ${ }^{40,41}$ whence under those conditions $P_{0}{ }^{a}$ is positive for an asymptotically empty and flat spacetime in the sense of reference 36 . (This positivity is not related to an intrinsic arrow of time, for the 4-vector $\boldsymbol{P}_{0}{ }^{\mathrm{a}}$ is defined relative to a conventional time-orientation of spacetime such that its positivity is preserved under a switch of time-orientation.) However, in contrast to a positivity statement about $\boldsymbol{P}_{+}{ }^{a}$, the positivity of the ADM-energy or even of $\boldsymbol{P}_{0}{ }^{\mathrm{a}}$ does not, by itself, imply restrictions about the energy that can be emitted in the form of gravitational waves.

In order to draw physically useful conclusions from the constancy (hypersurface independence) and positivity of $\boldsymbol{P}_{0}{ }^{a}$ it appears to be necessary to decompose, for "late" Cauchy hypersurfaces when radiation has travelled away from its sources, the total $P_{0}{ }^{2}$ into summands assignable to the source and the radiation, respectively, and to establish positivity separately for these parts. The possibility of such a procedure is

\footnotetext{
** We call a 4-vector positive if it is contained in the interior of the future null half cone. We assume spacetime to be time-oriented.
} 
suggested by a formula ${ }^{42}$ analogous to $(6)$ for $P_{0}{ }^{a}$, but I am unaware that it has been carried out.

Related to the problem just mentioned is the question of the connection between the Dixon 4-momenta $p^{\mathrm{a}}$ of the bodies considered in the previous section and the asymptotic quantities $P_{0}{ }^{a}, P_{+}{ }^{a}$. Expressions of $P_{0}{ }^{a}$ and /or $P_{+}{ }^{a}$ in terms of the $p^{a}$ and field contributions would seem to be needed to relate the asymptotic energymomentum balance (5) to the motion of the bodies and, thus, to observable properties of isolated systems.

The problem of defining asymptotic angular momenta, to establish for them a law analogous to (5) etc. has been considered, but will not be discussed here. (See references $12,36,39,43$.)

Whereas future null infinity is used to describe outgoing radiation, incident radiation can be described naturally in terms of asymptotic fields on past null infinity, $\mathfrak{I}^{-}$. The analog of equation 5 for $\mathcal{I}^{-}$shows: The condition

$$
\underline{P}^{\mathrm{a}}=\mathrm{const}
$$

which implies $P^{\mathrm{a}}=P_{0}{ }^{\mathrm{a}}$ ) is necessary and sufficient for absence of incoming gravitational radiation. This condition is equivalent to the vanishing of the advanced time-derivative of the asymptotic shear at $\mathfrak{J}^{-}$. (For these and alternative conditions see references 39,44 .)

For asymptotically empty and flat spacetimes the metric, curvature, etc. can be represented, in a neighborhood of null infinity (far radiation zone), by asymptotic series in inverse powers of a radial coordinate, with coefficients depending on angles and retarded (or advanced) time; see, e.g. reference 45 and the references therein.

I conclude this section with some critical remarks taken partly from B.G. Schmidt. ${ }^{35}$ The concepts of asymptotic flatness have been invented to describe the gravitational field of isolated systems far away from the sources. The main motivations came from linearized theory, stationary exact solutions and peeling properties of algebraically special vacuum fields. Since neither an exact solution of Einstein's field equation describing a bounded source emitting gravitational radiation nor even an existence proof for such a solution is known, the definitions of asymptotically flat spacetimes given so far have to be considered as proposals, the adequacy of which, for their intended purpose, is open to questions and needs to be investigated further. In particular, it is not known whether "typical" radiation fields fall off, and sources behave sufficiently quietly in the distant past, in order that a null- or space-like infinity exists and/or has the differentiability which has been assumed (conjectured) in currently used definitions of asymptotic flatness. Some perturbation calculations on scalar, massless waves in Schwarzschild spacetime by B.G. Schmidt and J.M. Stewart ${ }^{46}$ and computations on the relativistic Kepler problem by $M$. Walker and C.M. Will ${ }^{47}$ indicate that the usual differentiability requirements at 9 are, in fact, too strong even for simple examples which belong to the intended domain of application of the notion of asymptotic flatness. In this connection it may also be appropriate to remember that it is not known, in spite of extensive work on the initial value problem for Einstein's equation, whether the maximally developed spacetime ${ }^{48,49}$ belonging to asymptotically flat initial data has even a piece of null infinity, and whether Cauchy data associated with boosted Cauchy hypersurfaces ${ }^{36}$ are asymptotically flat.

It seems important to keep in mind that asymptotics is not a theory for its own 
sake, but a tool for analyzing and, I hope, constructing models of isolated systems and deriving properties of them. As a tool, it is important in so far as it serves these purposes. Since exact models of radiating systems are not in sight, it appears to be urgent to connect the concepts and constructions of asymptotics with approximation methods, even if that requires sacrificing some of its geometrical beauty or modifying its assumptions.

\section{ApProximation Methods AND Equations of Motion}

Attempts to construct relativistic models of isolated systems containing more than one body have been made since the beginning of general relativity by means of formal, analytical approximation methods and, only recently, by numerical methods. For the latter, see L. Smarr ${ }^{50}$ and the contribution by D. Eardley to these proceedings.

In this section I shall outline a new, improved version of a weak-field, slow-motion approximation method; indicate results which have been obtained by means of it; discuss its drawbacks and suggestions to overcome them; and make some remarks about alternative methods. A general review of approximation methods will not be attempted here.

The approximation method to be outlined is a modification, proposed and sketched by myself ${ }^{4}$ and elaborated by D. Kerlick ${ }^{51}$ and A. Caporali, ${ }^{52}$ of the method of J.L. Anderson and T.C. Decanio. ${ }^{53}$ Theirs as well as the new method incorporate ab initio Fock's no-incoming-radiation-condition ${ }^{24,44}$ into a systematic iteration scheme which uses the harmonic gauge throughout, in contrast to the method of Chandrasekhar et al. ${ }^{54}$ The new method is, at least formally, an improvement in two respects: 1) All approximate metric components, Christoffel symbols etc. needed to set up the $2 \frac{1}{2}$ post-Newtonian equations of motion are finite; no divergent expressions occur during the calculations leading up to those equations. 2) The components of the metric and the connection at some coordinate time are obtained as functionals of the Newtonian Cauchy data (mass density and coordinate 3-velocity of matter) pertaining to that same instant. Hence the post-Newtonian forces in the equations of motion do not contain time derivatives. Thus, initial data for the matter variables determine, at least formally, a unique solution, and classical perturbation theory can be applied directly. On the other hand, the new method does give divergent terms in the third PNA. (This will be discussed below.)

The new method is based on the reduced Einstein equation

$$
g^{\mathrm{cd}} \partial_{\mathrm{cd}} g^{\mathrm{ab}}+Q^{\mathrm{ab}}(g, \partial g)=2(-\mathrm{g}) T^{\mathrm{ab}},
$$

obtained by writing equation (1) in terms of the contravariant metric density $g^{\text {ab }}:=$ $\sqrt{-g} g^{\mathrm{ab}}\left(g:=\operatorname{det} g_{\mathrm{ab}}=\operatorname{det} g^{\mathrm{ab}}\right)$ and omitting terms containing $g^{\mathrm{ab}} \mathrm{b}$. Clearly, a solution of (8) obeys (1) provided the harmonic gauge condition

$$
g^{\mathrm{ab}}, \mathrm{b}=0
$$

is also satisfied. One now chooses an auxiliary flat Lorentz metric $\eta^{\text {ab }}$ and defines the metric deviation

$$
k^{\mathrm{ab}}:=g^{\mathrm{ab}}-\eta^{\mathrm{ab}}
$$


There is no invariant measure of the "smallness" of $k^{\mathrm{ab}}$ at a point (E. Eder ${ }^{55}$ has shown that no natural distance function exists on the set of Lorentzian metrics on a vector space.) For the following approximation scheme in which $k^{\text {ab }}$ is computed first and (11) is then used to find $g^{\mathrm{ab}}, g_{\mathrm{ab}}$ etc., it is sufficient to assume that for $0 \leq x \leq 1$, det $\left(\eta^{\mathrm{ab}}+x k^{\mathrm{ab}}\right)<0$; let the set of these $k^{\text {ab }}$ be denoted as $\AA$. (This mild weak-field assumption holds even for neutron stars.) One verifies easily:

1) $g^{\mathrm{ab}}=\eta^{\mathrm{ab}}+k^{\mathrm{ab}}$ is Lorentzian if $k^{\mathrm{ab}}$ is in $\Re$.

2) $(-g),(-g)^{-1 / 2}, g_{\mathrm{ab}}$ and $g^{\mathrm{ab}}$ are analytic functions of $k^{\mathrm{ab}}$ on $\AA$.

3) The partial derivatives $(-g)_{\kappa_{c}}, \ldots, g^{\text {ab }}{ }_{{ }_{c}}$ are (in an obvious sense) analytic functions of $k^{\mathrm{ab}}$ and $k^{\mathrm{ab}}{ }_{{ }_{\mathrm{c}}}$ provided $k^{\mathrm{ab}}$ is in $\AA$. Analogous statements hold for higher derivatives and, therefore, for $\Gamma_{\mathrm{bc}}{ }^{\mathrm{a}}, R_{\mathrm{ab}}$ etc.

One now works in orthonormal coordinates with respect to $\eta^{\text {ab }}$ so that (10) can be replaced by

$$
k_{, b}^{\mathrm{ab}}=0,
$$

and rewrites $(\mathbf{8})$ (modulo (10)) as

$$
\frac{1}{2} \square k^{\mathrm{ab}}=g\left(T^{\mathrm{ab}}+t^{\mathrm{ab}}\right)+\left(k^{\mathrm{a}(\mathrm{b}} k^{\mathrm{d} \mathrm{c} \mathrm{c}}\right), \mathrm{cd}
$$

where $\left[:=\vec{\nabla}^{2}-\partial_{\mathrm{u}}\right.$ is the flat-space wave operator. In $\AA$, the Landau-Lifshitz energy-momentum pseudo-tensor $t^{\text {bb }}$ of the gravitational field depends analytically on $k^{\mathrm{ab}}$ and its first derivatives. The last term on the r.h.s. of (13) contains even second derivatives of $k^{\text {ab }}$; its divergence vanishes identically. Hence, (12) and (13) imply the (nontensorial) conservation law

$$
\left.1-g\left(T^{\mathrm{ab}}+t^{\mathrm{ab}}\right)\right\}_{b}=0 .
$$

Choose as a source model an isentropic perfect fluid,

$$
T^{\mathrm{ab}}=(\rho+p) U^{\mathrm{a}} U^{\mathrm{b}}-p g^{\mathrm{ab}}, \quad p=p(\rho), \quad|p|<\rho,
$$

and define as matter variables the density $\rho$ and the coordinate 3-velocity $\vec{v}:=$ $\left(U^{\lambda} / U^{0}\right)$. Because of $g_{\mathrm{ab}} U^{\mathrm{a}} U^{\mathrm{b}}=1$ and $(11), U^{0}$ and $T^{\mathrm{ab}}$ can be considered as functions of $\rho, \vec{v}=\left(v^{\wedge}\right)$ and $k^{\mathrm{ab}}$;

$$
T^{\mathrm{ab}}=T^{\mathrm{ab}}\left(\rho, \overrightarrow{\boldsymbol{v}}, k^{\mathrm{cd}}\right)
$$

The last condition in (15) implies that $T_{\mathrm{b}}{ }^{2}$ satisfies the energy condition formulated in the section entitled "Bodies and Laws of Motion."

In view of the remarks made after equation 11 , equation 13 is of the form

$$
\begin{aligned}
-\frac{1}{2} \square k= & \left(1+f_{1}(k)\right) T(\rho, \vec{v}, k)+f_{2}(k, \partial k) \\
& +f_{2}\left(k, \partial k, \partial^{2} k\right)=: 2 \pi \Delta .
\end{aligned}
$$

Here tensor indices have been suppressed, and $f_{v}$ denotes a (numerical or matrix valued) analytic function of $k$ and the indicated derivatives which starts with terms of order $\nu$ in $k$ and its derivatives.

If $k$ satisfies Fock's condition for absence of incoming radiation, ${ }^{24.44}$ 


$$
k=o(1), \quad\left(\frac{\partial}{\partial r}+\frac{\partial}{\partial t}\right) k=o\left(r^{-1}\right)
$$

uniformly in neighborhoods of past-directed, flat-space null cones, then (17) implies, via Kirchhoff's identity, the integro-differential equation

$$
\left.k \overrightarrow{(\mathbf{x}, t)}=\int_{\Re^{3}} \Lambda \overrightarrow{(\mathbf{x}}+\overrightarrow{\mathbf{y}}, t-\overrightarrow{|\vec{y}|}\right) \frac{d^{3} y}{|\vec{y}|}
$$

Postponing objections against (18), let us proceed with the algorithm. One now formally Taylor-expands the integrand with respect to its time argument at $t$, obtaining

$$
k=\sum_{n=0}^{\infty} \frac{(-1)^{n}}{n !} I_{n-1}\left(\partial_{1}{ }^{n} \Delta\right)
$$

where

$$
I_{\mathrm{n}}(f)(\overrightarrow{\boldsymbol{x}}, t):=\int f(\overrightarrow{\boldsymbol{x}}+\overrightarrow{\boldsymbol{y}}, t)|\overrightarrow{\boldsymbol{y}}|^{\mathrm{n}} d^{3} y .
$$

The right hand side of equation 20 contains time derivatives of all orders due to the retardation expansion, just as the field expansion in Lorentz's electron theory. Convergence of the series in (20) is neither to be expected (except for stationary situations) nor relevant; it is part of a formal algorithm.

One can now carry out the following iteration: ${ }^{4,51}$ Put $k=0$ in $\Lambda$ and in equation 14. Define, guided by (20), $k^{\text {ab }}$ by

(1)

$$
\begin{aligned}
& \underset{(1)}{k^{0 \mathrm{a}}}:=I_{-1}\left(\Lambda^{0 \mathrm{a}}\right)-I_{0}\left(\delta_{2} \Lambda^{0 \mathrm{a}}\right) \\
& \text { (1) }^{k^{\lambda \mu}}:=0 \text { for } \lambda, \mu=1,2,3 \text {, }
\end{aligned}
$$

and eliminate $\dot{\rho}, \vec{\nu}$ by means of $(14)_{k-0}$, to obtain $k^{\mathrm{ab}}$ as a functional of $\vec{\rho}$ and $\vec{\nu}$.

Then, insert $k^{\mathrm{ab}}$ into $\Lambda$ (equation 17) and (14) and compute $k^{\mathrm{ab}}$ from (20), keeping (1) for each component two more terms of the series than were used in (22), and eliminate time derivatives of $\rho$ and $\vec{v}$ by means of the "improved" equation 14 , to obtain $k^{\text {ab }}$ as a functional of $\rho, \vec{v}$ and their spatial derivatives. Continue in this way to obtain $k^{a b}$ etc. $\dagger \dagger$

If the density $\rho$ has compact support this procedure gives convergent integrals for $\underset{(1)}{k^{\mathrm{ab}}}$ and $\underset{(2)}{k^{\mathrm{ab}}}$. Most contributions to $\underset{(3)}{k^{\mathrm{ab}}}$ are also well-defined, but some of its terms


diverge. ${ }^{51}$ To order the various terms in $\underset{(v)}{k^{\mathrm{ab}}}, \underset{(\nu)}{T^{\mathrm{ab}}}:=T^{\mathrm{ab}}\left(\rho, \overrightarrow{\mathrm{v}}, \underset{(v)}{\mathrm{k}^{\prime \prime}}\right)$ and the expressions derived from them, like $\Gamma_{\mathrm{bc}}{ }^{\mathrm{a}}$ etc, one can either keep the speed of light $c$ in (v)

††This algorithm to obtain $k, k$ etc. is neither a formal power series expansion nor an (1) (2)

iteration in the usual sense. It is similar to a Hilbert expansion used to obtain so-called "normal" asymptotic series solutions of Boltzmann's integro-differential equation. 
all equations and order terms according to powers of $c^{-1}$, or use physical arguments to assign orders of magnitude to $\rho, \overrightarrow{\mathrm{v}}, \mathrm{d} p / \mathrm{d} \rho$ etc. to obtain a dimensionless small parameter for this purpose.

If $k^{\mathrm{ab}}$ is in $\Re$, it determines a Lorentz metric $g^{\mathrm{ab}}$. There is no reason for the pair $\left(g_{\mathrm{ab}}, T^{\mathrm{ab}}\right)$ to obey Einstein's equation approximately. But if one imposes on such a pair (N) $(\mathrm{N})$

an approximate equation of motion by inserting $g_{\mathrm{ab}}, T^{\mathrm{ab}}$ into the local law of motion (N) (N)

(2) and keeping consistently terms up to some assigned order of magnitude, or, alternatively, imposes the conservation law (14) or the gauge condition (12), then one might expect-from the way the algorithm has been set up-that those solutions of the approximate equations of motion which describe slowly moving bodies with small "compactness" ( = Schwarzschild radius/geometrical radius), do indeed give approximate solutions of $(\mathbf{1})$ in a near zone containing the bodies, small compared to a wavelength characteristic of the source motion. This expectation is supported, at least for the first post-Newtonian approximation, by some plausible estimates ${ }^{52}$ of the "error tensor" $\mathrm{E}_{\mathrm{ab}}:=\boldsymbol{R}_{\mathrm{ab}}-\left(T_{\mathrm{ab}}-1 / 2 g_{\mathrm{ab}} T\right)$ associated with a pair $\left(g_{\mathrm{ab}}, T^{\mathrm{ab}}\right)$, but no exact error estimates are known.

(N) (N)

If the local, first post-Newtonian equations of motion (which are equivalent to those of Chandrasekhar ${ }^{22}$ and Synge ${ }^{56}$ ) are integrated over the bodies, and if these bodies are assumed to be well-separated, nearly spherical, and nonrotating, then one recovers for the center-of-mass motions the Einstein-Infeld-Hoffmann equations, in accordance with older results, provided one identifies the positions and masses of EIH with suitable quantities defined within the $1 \mathrm{PN}$-framework. ${ }^{52,57}$ (In connection with this derivation, and in view of the remarks about the self field problem, the following may be of interest. If, within the IPNA, one subtracts the self potential of a body from the total potential, then because of the nonlinear dependence of the potential on the source variables, the resulting difference potential still depends on the body under consideration, and the self potential depends on the other bodies too. What distinguishes the self potential of a body from the difference potential is that, within that body, the former varies on the scale of the size of the body, whereas the latter varies slowly, on the scale of the distance to the other bodies. This should be valid also in the exact theory, as emphasized by Dixon. ${ }^{17}$ ) If one assumes the bodies to be axially symmetric and rotating, one obtains in addition the spin-precession formulae (Caporali and Spyrou, to be published) derived previously by different, in my opinion, less satisfactory methods. ${ }^{58-60}$

As far as I am aware, the second post-Newtonian, local hydrodynamic equations of motion have not been used to obtain equations of motion for bodies as a whole.

Being in possession of the improved, infinity-free $21 / 2$ PN local equations of motion derived by Kerlick, one can repeat the Chandrasekhar-Esposito ${ }^{54}$ analysis to identify radiation damping force densities, and set up a near-zone energy-momentum balance which can then, hopefully, be connected with the asymptotic balance (5) as indicated in FIGURE 1. This work has not yet been completed.

Let us now look at the approximation method critically. A first question mark should be attached to equation 10 or 12 , respectively. It has not been shown that harmonic coordinates exist globally, as was assumed above. Although Y. ChoquetBruhat, D. Christodoulou and $M$. Francaviglia ${ }^{61}$ have recently shown that such coordinates do exist globally prior to some Cauchy-hypersurface if the metric is in a 


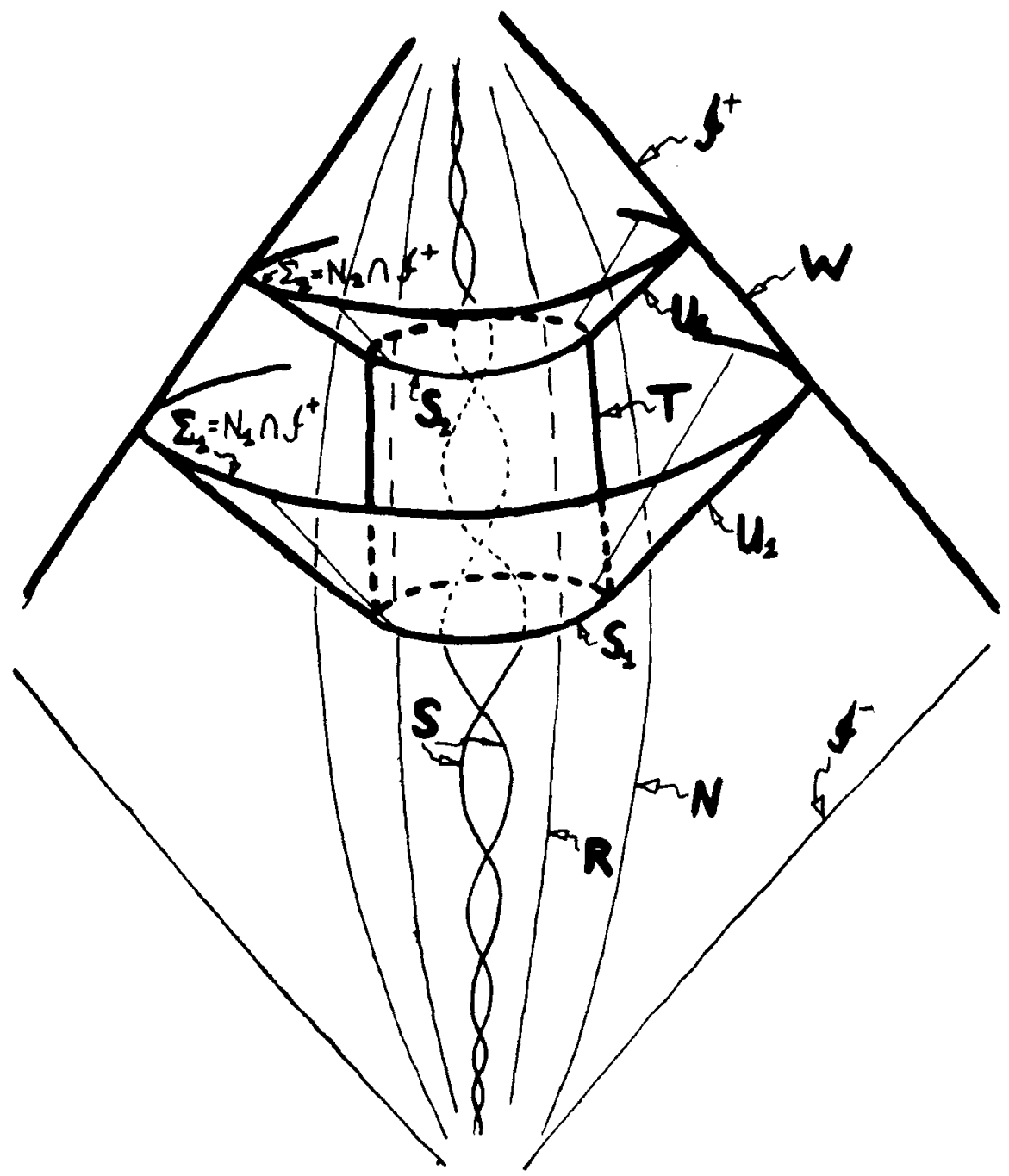

FIGURE 1. Connection between near-zone and asymptotic energy-momentum balances. Outside $R$ is the radiation zone, inside $N$ the near zone containing the source $S . S_{1}$ and $S_{2}$ are oriented, spacelike cross sections of the near zone; $T$ is a timelike cylinder connecting the spherical boundaries of $S_{1}, S_{2}$, respectively; $U_{1}, U_{2}$ are outgoing null hypersurfaces; and $W$ is the part of future null infinity which is between the cross sections $\Sigma_{1}$ and $\Sigma_{2} . P\left(S_{1}\right)$ etc. denotes the 4-momentum flux of the Landau-Lifshitz energy-momentum complex through $S_{1}$ etc. (as in equation 6 of the text), computed in a suitable, approximately orthonormal coordinate system. Then the inner balance is $P\left(S_{2}\right)-P\left(S_{1}\right)=-P(T)$; it can be approximately evaluated by means of the near-zone approximation, provided the $S_{\mathrm{i}}$ are chosen suitably. This relation is substantially equation 110 in reference 22, interpreted geometrically. The outer balance is $P(W) \approx P(T)$, since $^{62} P\left(U_{\mathrm{i}}\right) \approx 0$ for outgoing radiation. The asymptotic balance (text, equation 5 ) is $P(W)=$ $P_{\mathrm{R}}\left(\Sigma_{1}, \Sigma_{2}\right)=$ Bondi-flux. In summary, then, $\left(P\left(S_{2}\right)-P\left(S_{1}\right)\right)_{\text {near zone }} \approx-\left(P_{\mathrm{R}}\left(\Sigma_{1}, \Sigma_{2}\right)\right)_{\text {uympl. }}$. This sketch indicates how one may be able to obtain an "overall balance," provided one carries out several estimates, defines suitable coordinates, etc. Such a reasoning should also provide inequalities to define a near zone and a radiation zone; it has not been carried out, as far as I am aware. 
certain sense asymptotically stationary in the infinite past, this still does not seem to cover generic cases of physical interest. Secondly, and more importantly, condition (18) in harmonic coordinates may not express absence of incoming radiation correctly; for, as is well known, already in the Schwarzschild spacetime the obvious splitting (11) with respect to harmonic coordinates leads to flat-space, past-directed null cones which are not asymptotic to the correct null cones, but which deviate from them logarithmically and terminate not on $\mathfrak{I}^{-}$but at spatial infinity, $i^{0}$; and even along the curved-space, past-directed null rays, the $\mathbf{k}^{\mathrm{ab}}$ do not seem to obey the conditions ${ }^{9}(\mathbf{1 8})$. (This point needs further clarification.) Moreover, using (13) or (19) one propagates all higher-order fields along the wrong null cones. Since the last term in equation 13 is the effective source contribution which compensates for the use of the wrong wave operator, and it is this term which leads to the lowest-order divergences in the modified slow-motion method, it appears to be necessary to relax the harmonic condition (10), and either to improve the splitting (11) at each step of the iteration, or to propagate the $(N+1)$ th-order field by means of the curved-space, $(N$-th $)$-order wave operator. ${ }^{63}$ It is not clear whether any improvement will leave the results up to the $2 \frac{1}{2}$ PN order intact, so doubt remains concerning their validity.

The use of the wrong propagation is, of course, a shortcoming also of the standard "fast-motion," Lorentz-invariant approximation methods. ${ }^{31,64}$ By combining a convergent iteration with one using "the wrong propagation," D. Christodoulou and B.G. Schmidt ${ }^{65}$ were able to prove that the latter iteration, although it in general diverges, nevertheless leads to an asymptotic approximation. Useful and exact estimates to justify a truncated retardation expansion $(\mathbf{1 9}) \rightarrow(20)$ are unknown to me.

So far, it appears to me that use of matched asymptotic expansions ${ }^{65}$ has not led to more concrete results or more exact derivations in the context of isolated systems than the more conventional method outlined above, although the underlying idea is attractive.

A. Rosenblum ${ }^{64}$ has recently calculated the energy loss due to emission of gravitational radiation during small angle scattering, using a fast-motion analog of the first post-Newtonian approximation. Since he treated the bodies as points, he had to regularize infinite, linear self field-terms, and he had to assume the existence of higher-order regularization procedures, though not their form. His result differs by a factor of about 2.3 from the one obtained by means of Einstein's quadrupole formula. According to this calculation the contributions due to the nonlinear, second-order terms of the metric are numerically comparable to those from the linear approximation. This result may cast some light on the antidamping obtained long ago by S.F. Smith and P. Havas ${ }^{67}$ on the basis of a linear, fast approximation calculation in which, perhaps, nonlinear terms would over-compensate the linear ones. However, the bound state problem has not been treated yet by a fast method beyond the linear stage. Rosenblum's result accentuates the need for further work to clarify the gravitational radiation damping problem.

\section{SUMMARY}

The first post-Newtonian approximation that includes the EIH-equations has been obtained by different formal approximation methods. The results agree; there do not appear to be serious objections against its validity. Thus the theoretical basis for the 
four classical tests of GR and the spin precession effects, for bodies of arbitrary compactness and for black holes, seems to be firm. A corresponding statement cannot be made, in my opinion, for the higher approximations, particularly not for gravitational radiation reaction effects, for reasons which I have discussed.

Even at the IPN level a connection of the approximation schemes with intrinsic, coordinate-free formulations would seem to be desirable for understanding, if not for computing effects. The basic problem of rigorously justifying the formal approximation is perhaps outside the reach of present mathematics.

Can a combination of approximation methods with exact approaches help to overcome the difficulties and unclarities discussed above, and to obtain answers to "concrete" questions? Can one formulate questions pertaining to, say, "seculas effects of the emission of radiation on the motion of binary systems," by means of intrinsic concepts of Einstein's theory without reference to approximation methods, and compute such effects by methods which do not use assumptions contradicting the underlying precise formulation? Can the recent work on the dynamics of GR, i.e. the evolution of Cauchy date, help to improve approximations? Maybe at one of the future Texas symposia we shall get some answers.

\section{REFERENCES}

1. Havas, P. 1973. In Ondes et Radiations Gravitationelles. Coll. Int. CNRS No. 220: 383. Paris.

2. Papapetrou, A. 1974. Lectures on General Relativity. Reidel. Dordrecht, Holland.

3. Ehlers, J., A. Rosenblum, J. N. Goldberg \& P. Havas. 1976. Ap. J. $208:$ L77.

4. EhLerS, J. 1977. In Proceedings of the International School of General Relativistic Effects in Physics and Astrophysics. Institute-Report MPI-PAE/Astro 138; 45. Munich.

5. Einstein, A. 1918. Sitzungsber. Preuss. Akadem. Wiss., Physik.-Math. K1.: 154.

6. Peters, P. C. 1964. Phys. Rev. 136: 1224.

7. WAGONER, R. V. 1975. Ap. J. Lett. 196: L63.

8. Trautman, A. 1958. Lectures on General Relativity. (Mimeographed notes). King's College. London.

9. IsaAcson, R. A. \& J. Winicour. 1968. Phys. Rev. 168: I45I; Madore, J. 1970. Ann. Inst. Henri Poincaré 12: 285, 365.

10. Dixon, W. G. \& J. M. BiRD. 1975. Ann. Phys. (N.Y.) 94: 320; Bird, J. M. 1976. Ann. Phys. 101: 345.

11. Geroch, R. P. 1977. In Asymptotic Structure of Spacetime. F. P. Esposito \& L. Witten, Eds.: 1. Plenum Press. New York, N.Y.

12. EhLeRS, J., Ed. 1979. Isolated Gravitating Systems in General Relativity. North-Holland Publ. Company. Amsterdam.

13. HawkING, S. W. 1968. J. Math. Phys. 9: 598.

14. Aichelburg, P. C. \& R. Beig. 1976. Ann. Phys. 98: 264; BeIG, R. In ref. 12:307.

15. Bondi, H., M. G. J. van Der Burg \& A. W. K. MetzNer. 1962. Proc. Roy. Soc. A269: 21; Sachs, R. K. 1962. Proc. Roy. Soc. A270: 103; Penrose, R. 1965. Proc. Roy. Soc. A284: 159.

16. INFELD, L. \& A. SCHILD. 1949. Rev. Mod. Phys. 21: 408.

17. DiXoN, W. G. 1979. Ref. 12: 156. See also the papers cited therein.

18. EhLERS, J. \& E. RUdOLPH. 1977. Gen. Rel. Grav. 8: 197.

19. D'Eath, P. D. 1975. Phys. Rev. D11: 1387.

20. Dirac, P. A. M. 1961. Lecture at the Intern. Conference on General Relativity and Gravitation held in Jablonna, Warsaw.

21. BoNDI, H. 1964. In Lectures on General Relativity. A. Trautman, F. A. E. Pirani, H. Bondi, Eds.: 375. Prentice-Hall. Englewood Cliffs, New Jersey. 
22. Chandrasekhar, S. 1970. In Relativity. M. Carmeli \& S. I. Fickler, Eds.: 81. Plenum Press. New York, N.Y.

23. Künzle, H. P. 1972. Ann. Inst. Henri Poincaré. 17: 337.

24. Fock, V. 1959. The Theory of Space, Time and Gravitation. Pergamon Press. London.

25. Dautcourt, G. 1964. Acta Physica Polonica 25: 637; Künzle, H. P. 1976. Gen. Rel. Grav. 7: 445.

26. EhLERS, J. 1979. Festschrift für P. Mittelstaedt. To be published.

27. Gibions, G. W. 1974. Commun. Math. Phys. 35: 13.

28. D'EATH, P. D. 1975. Phys. Rev. D12: 2183.

29. SChattner, R. 1979. Gen. Rel. Grav. 10: 377, 395.

30. SChattner, R. 1979. Ph.D. Thesis. University of Munich. To be published.

31. Havas, P. \& J. N. Goldberg. 1962. Phys. Rev. 128: 398.

32. WeYL, H. 1923. Raum, Zeit, Materie (5th ed.). Springer Verlag. Berlin.

34. SChмidT, B. G. 1977. See ref. 11: 429.

35. SCHMIDT, B. G. 1979. In ref. 12: 11.

36. Ashtekar, A. \& R. O. HaNSEN. 1978. J. Math. Phys. 19: 549.

37. Ashrekar, A. \& A. Magnon-Ashtekar. 1979. Preprint. Université de Clermont-Fd. 63170 Aubière, France.

38. SACHS, R. K. 1962. Phys. Rev. 128: 2851.

39. Streubel, M. 1978. Dissertation. University of Hamburg. (Available as report MPIPAE/Astro 165 from Max-Planck-Institut für Physik und Astrophysik, Munich.)

40. Chopuet-Bruhat, Y., A. E. Fischer \& J. E. Marsden. 1979. In ref. 12.

41. SCHOEN, R, \& S. T, YAu. 1979. Commun. Math. Phys. 65: 45.

42. Misner, C. W., K. S. Thorne \& J. A. Wheeler. 1973. Gravitation. Freeman and Comp. San Francisco.

43. Winicour, J. 1968. J. Math. Phys. 9: 861.

44. LeIPOLD, G. \& M. WALKer. 1977. Ann. Inst. Henri Poincarè 27: 61.

45. Exton, A., E. T. Newman \& R. Penrose. 1969. J. Math. Phys. 10: 1566; Ludwig, G. 1976. Gen. Rel. Grav. 7: 293.

46. SChmidT, B. G. \& J. M. STEWART. 1978, Report MPI-PAE/Astro 161 of the MPI (see reference 39 ).

47. WALKER, M. \& C. M. WILl. 1979. Phys. Rev. D19: 3483, 3495.

48. Choquet-Bruhat, Y. \& R. P. Geroch. 1969. Comm. Math. Phys. 14: 329.

49. Hawking, S. W. \& G. F. R. Ellis. 1973. The Large-Scale Structure of Spacetime. University Press. Cambridge.

50. SMARR, L. 1977. Ann. N.Y. Acad. Sci. 302: 569.

51. KerLICK, D. To be published in Gen. Rel. Grav.

52. Caporali, A. 1979. Ph.D. Thesis. University of Munich. To be published.

53. Anderson, J. L. \& T. C. Decanio. 1975. Gen. Rel. Grav. 6: 197.

54. Chandrasekhar, S. \& F. P. Esposito. 1970. Ap. J. 160: 153.

55. EDER, E. 1979. Report MPI-PAE/Astro 186 of the MPI (see reference 39).

56. Synge, J. L. 1970. Proc. Roy. Irish Acad. 69A: 11; MCCreA, J. D. \& G. O'Brien. preprint DIAS-TP-78-23. Dublin.

57. Hogan, P. A. \& J. D. MCCrea. 1974. Gen. Rel. Grav. 5: 79; Spyrou, N. 1978. Gen. Rel. Grav. 9: 519 .

58. Barker, B. M. \& R. F. O'Connell. 1975. Phys. Rev. D12: 329.

59. Börner, G., J. Ehlers \& E. Rudolph. 1975. Astron. Astrophys. 44: 417.

60. McCreA, J. D. \& G. O'Brien. 1978. Gen. Rel. Grav. 9: 1101.

61. Chopuet-Bruhat, Y., D. Christodoulou \& M. Francaviglia. In preparation.

62. ISAACSON, R. A. 1968. Phys. Rev. 166: 1263, 1272.

63. THORNE, K. S. \& S. J. KOVÁCZ. 1975. Ap. J. 200: 245.

64. Rosenblum, A. 1978. Phys. Rev. Lett. 41: 1003.

65. Christodoulou, D. \& B. G. SChmidT. 1979. Commun. Math. Phys. 68: 275.

66. BURKe, W. L. 1979. In ref. 12: 220; also 1971, J. Math. Phys. 12: 401.

67. SMith, S. F. \& P. Havas. 1965. Phys. Rev. 138: 495.

68. MANSSON, B. 1978. Gen. Rel. Grav. 9: 1049. 